\title{
El emprendimiento educativo y el centro de lecto escritura académica de la Universidad Politécnica Salesiana, sede Guayaquil
}

\section{The educational undertaking and the academic writing center of the Salesian Polytechnic University, Guayaquil}

Marjorie Isabel Salgado Ruiz

Universidad Politécnica Salesiana, Ecuador

Autor corresponsal: marjoriesalgado55@hotmail.com

Fecha de recepción: 30 de Septiembre de 2017 - Fecha de aceptación: 15 de Abril de 2018

Resumen: Este trabajo valora los resultados de una estrategia de trabajo educativo para fortalecer las competencias comunicativas de los estudiantes universitarios como futuros emprendedores, a través de las actividades de una institución de marcada significación dentro de la universidad, denominada Centro de Lecto Escritura Académica (CLEA) de la Universidad Politécnica Salesiana, sede Guayaquil en el período 2016-2017. Precisamente el objetivo general del CLEA se dirige a fortalecer las competencias de lectura y escritura en los estudiantes de la Universidad Politécnica Salesiana y constituirse en un centro de apoyo a la consolidación de las habilidades linguísticas como eje transversal del proceso enseñanza-aprendizaje de las ofertas académicas del nivel de grado y posgrado, favoreciendo el acceso, permanencia y egreso exitoso de los estudiantes. Mediante la utilización de una metodología eminentemente cualitativa se aplicó una combinación de métodos y técnicas de investigación, que permitieron llegar a resultados positivos. Junto con métodos teóricos como el análisis y la síntesis, la inducción y la deducción y el estudio comparativo, se pusieron en práctica técnicas empíricas asociadas al trabajo en grupo, la entrevista y el análisis documental. Los resultados del trabajo incluyeron la ejecución de variadas actividades en el marco del proceso docente de las asignaturas curriculares, así como la realización de conversatorios literarios a nivel de la institución, con la participación de estudiantes y profesores de las diversas carreras universitarias. Particular significado se expresó en las tareas planificadas y en la ejecución de un cronograma de trabajo ejecutado por los integrantes del CLEA. Ello demostró la importancia de los procesos de la lecto escritura en la consecución de resultados en esta dirección, así como el incremento de la motivación lectora en los educandos. Se concluye que el empleo de variadas motivaciones y la aplicación de estrategias de aprendizaje durante el proceso educativo permiten mejorar las habilidades lingüísticas de los estudiantes, como futuros emprendedores en diversos campos de las tecnologías y las humanidades.

Palabras Claves: lecto escritura; estrategias; competencias comunicativas; habilidades linguiísticas; proceso de enseñanza-aprendizaje

Abstract: This work evaluates the results of an educational work strategy to strengthen the communicative competences of university students as future entrepreneurs, through the activities of an institution of great significance within the university, denominated 'Centro de LectoEscritura Académica’ (CLEA) of the Universidad Politécnica Salesiana, Guayaquil headquarters 
in the period 2016-2017. Precisely, the general objective of the CLEA is to strengthen reading and writing skills in students at this university, and to become a support center for the consolidation of language skills as a transversal axis of the teaching-learning process of academic offerings of the level of degree and postgraduate, favoring the access, stay and successful exit of the students. The use of an eminently qualitative methodology applied a combination of research methods and techniques, which allowed for positive results. Along with theoretical methods such as analysis and synthesis, induction and deduction, and comparative study, empirical techniques associated with group work, interviewing and documentary analysis were put into practice. The results of the work included the execution of various activities within the framework of the teaching process of the curricular subjects, as well as the accomplishment of literary conversations at the level of the institution, with the participation of students and professors of the diverse university careers. Particular significance was expressed in the planned tasks and in the execution of a work schedule executed by the members of the CLEA. This demonstrated the importance of the processes of reading in the achievement of results in this direction, as well as the increase of reading motivation in learners. It is concluded that the use of varied motivations and the application of learning strategies during the educational process allow to improve the linguistic abilities of the students, as future entrepreneurs in diverse fields of the technologies and the humanities.

Key words: reading and writing; strategies; communicative skills; language skills; teachinglearning process

\section{Introducción}

Este trabajo se propone como objetivo valorar los resultados de una estrategia de trabajo educativo para fortalecer las competencias comunicativas de los estudiantes universitarios como futuros emprendedores, a través de las actividades de una institución de marcada significación dentro de la universidad, denominada Centro de Lecto Escritura Académica (CLEA) de la Universidad Politécnica Salesiana.

El debate sobre la utilidad del desarrollo de la Lecto escritura en la universidad ha condicionado la necesidad de profundizar en esta importante vertiente de la formación integral de los estudiantes universitarios como futuros emprendedores. La propia acción educativa pudiera concebirse como una gestión emprendedora, si se tiene en cuenta los conceptos más actualizados sobre el emprendimiento educativo. Lo cierto es que organismos internacionales como la UNESCO, plantean la necesidad de jerarquizar el trabajo educativo en torno al conocimiento de la expresión oral y escrita.

Propiciar actividades que conlleven al buen uso de la lengua en un centro de instrucción superior puede resultar una tarea compleja, cuando se valora la influencia creciente de las nuevas tecnologías de la Comunicación sobre los estudiantes. Otros factores también influyen en la complejidad del tema, si se consideran las limitaciones en el uso del lenguaje en la realización de las tareas estudiantiles, incluyendo los trabajos académicos. Si bien es cierto, que los propios usuarios de las redes sociales coinciden en la importancia de escribir correctamente, se continúa observando dicho problema como resultado del empleo deficiente de las nuevas herramientas tecnológicas y hasta como una identidad de los usuarios. 
Todo ello ha originado que las autoridades educativas y los docentes especializados o no en el idioma investiguen sobre las técnicas o estrategias que conduzcan al conocimiento de la normativa de la lengua materna, sobre todo desde su incidencia eminentemente práctica. El cuestionamiento sobre la utilidad del desarrollo de la Lecto escritura en la universidad ha condicionado la necesidad de profundizar en determinados referentes que propicien el sustento teórico para emprender acciones educativas de envergadura en las instituciones de Educación Superior. Algunos elementos fundamentales pueden posibilitar el acercamiento a esta problemática.

La concepción del trabajo educativo para el desarrollo de la Lectoescritura en la universidad, requiere del tratamiento de determinados puntos de vista más actuales sobre el emprendimiento educativo, la gestión pedagógica en las instituciones de Educación Superior y la Didáctica general con sus derivaciones más específicas relacionadas con la expresión oral y escrita y la Didáctica universitaria. (Vázquez, A., 2010)

En ese contexto, el emprendimiento educativo supone un esfuerzo notable en la aplicación de los preceptos educativos de la sociedad en su conjunto y de las instituciones escolares en particular. El contenido de la educación para el emprendimiento se asocia a la preparación del individuo para la vida, a partir de concebir la formación de los sujetos sociales con espíritu emprendedor, desde el desarrollo de conocimientos, habilidades, competencias, capacidades, hábitos, puntos de vista, convicciones, valores y otros elementos que hacen posible que los individuos actúen en correspondencia con las exigencias. (Rodríguez, I., 2015)

En ese sentido, resultan importantes las consideraciones sobre la Didáctica como la ciencia pedagógica debate la existencia de un objeto de estudio particular, centrado en el proceso de enseñanza y aprendizaje con sus complejidades actuales; las peculiaridades de los componentes didácticos y personales, así como el desarrollo de un sistema categorial propio, a partir de la evolución histórica del pensamiento didáctico desde Juan Amos Comenio, considerado por diversos autores como el "padre de la Didáctica", desde su obra cumbre "Didáctica Magna".

Igualmente, desde el punto de vista de la Didáctica de la Lectoescritura resultan relevantes las consideraciones de especialistas en el tema, que propugnan el aprendizaje activo de conocimientos, habilidades, valores y competencias sobre la lectura y la escritura. Para algunos autores se trata de un nuevo modelo de enseñar sustentado en "... ayudar a aprender conceptos y prácticas discursivas disciplinares por medio de tareas para reelaborar y adueñarse de unos y otras simultáneamente". (Carlino, P., 2005)

"La escuela se convierte en un espacio privilegiado para potenciar la tolerancia, la convivencia entre los pueblos, e inculcar el valor positivo de la diversidad, siendo la respuesta a la misma uno de sus retos fundamentales en el siglo XXI". (Azpillaga, V.,2011)

De igual forma, los textos permiten conocer otras formas de pensar, genera conocimientos y promueve la reflexión la cual desarrolla el sentido crítico. Siendo la literatura un aprendizaje a lo largo de la vida es imprescindible para la maduración personal e intelectual de los jóvenes. "Con la lectura de obras literarias se consigue el desarrollo del lenguaje que es de 
suma importancia en un mundo como el que estamos viviendo; porque en la medida que una persona sea más capaz de dominar la palabra será menos susceptible de manipulación." (Alonso, F.,2017).

Ya desde hace una década se ha reafirmado la significación de la lectoescritura en la formación de los educandos. "La Confederación Industrial Británica (2007) definió para el Reino Unido un conjunto de habilidades fundamentales para la empleabilidad incluyendo la comunicación, las relaciones con los clientes, el trabajo en equipo y la resolución de problemas, pero también, la capacidad de lecto-escritura, la competencia matemática y el uso de tecnologías." (Cobo, C.,2012). De igual forma se ha reconocido que “...lectura en general y la lectura de obras literarias en particular, como unos de los instrumentos más eficaces para la formación de la personalidad y el conocimiento profundo del mundo que nos rodea y de nosotros mismos". (Alonso, F.,2017)

Precisamente, el diagnóstico de la situación de los estudiantes en el domino de las cuatro habilidades básicas del idioma ha demostrado la existencia de serias insuficiencias, las que han sido críticas en algunos estudiantes de nivel de pregrado en determinadas carreras. Ello afecta sensiblemente el proceso de formación profesional de los futuros graduados. Esto se ha corraborado mediante la observación de los resultados de los exámenes de los estudiantes en las asignaturas del área de Humanidades y en particular en la expresión oral y escrita. Las entrevistas con docentes encargados de esas asignaturas reafirman la existencia de un déficit significativo en la escritura, la lectura, el escuchar y hablar correctamente.

De esa manera fue necesario plantearse interrogantes científicas que permitieran dirigir el proceso investigativo hacia la solución de las dificultades más notables. El principal cuestionamiento se planteó en los términos de ¿cómo contribuir a resolver las insuficiencias de los estudiantes en el conocimiento de aspectos fundamentales del idioma? A partir de lo anterior, el objetivo principal fue diseñar una estrategia de trabajo que facilitara la motivación por la lectoescritura como vía para contribuir a resolver las deficiencias de los estudiantes.

Para el cumplimiento del objetivo propuesto fue necesario realizar el bosquejo de la modelación del proceso de desarrollo de las habilidades comunicativas mediante la lectoescritura. En ese sentido se consideró que este proceso puede concebirse mediante la interacción de un conjunto de factores incidentes, tales como la gestión integrada de determinadas estructuras a nivel general de la IES o en las sedes respectivas, la determinación de los sujetos que participan en el proceso, la delimitación de los objetivos generales y específicos, así como la ejecución de un conjunto de acciones en esta dirección.

Este trabajo se realizó mediante la utilización de una metodología de investigación eminentemente cualitativa y para su realización se aplicó una combinación de métodos y técnicas de investigación para desarrollar la lectoescritura, que permitieron llegar a resultados positivos. Junto con métodos teóricos como el análisis y la síntesis, la inducción y la deducción y el estudio comparativo, se pusieron en práctica técnicas empíricas asociadas al trabajo en grupo, la entrevista y el análisis documental, que permitieron comprobar los logros y dificultades en el proceso. 


\section{Una estrategia para desarrollar la lectoescritura en la universidad}

La constitución del Centro de Lecto Escritura Académica, CLEA, en la sede de Guayaquil de la Universidad Politécnica Salesiana del Ecuador en el 2013 (Centro UPS., 2013), posibilitó poner en práctica importantes aspectos para la formación de los estudiantes en la lectura y la escritura académica. El objetivo general del centro se ha dirigido a fortalecer las competencias de lectura y escritura en los estudiantes y constituirse en un centro de apoyo a la consolidación de las habilidades lingüísticas como eje transversal del proceso enseñanzaaprendizaje de las ofertas académicas del nivel de grado y posgrado, favoreciendo el acceso, permanencia y egreso exitoso de los estudiantes. De igual manera, los objetivos específicos del CLEA se proponen generar espacios de formación para los estudiantes y docentes de las distintas carreras de la UPS, con proyección a fortalecer las habilidades de lectura y escritura para aplicarlas en las diferentes actividades del proceso enseñanza-aprendizaje. Igualmente, el CLEA pretende fomentar la redacción científica como parte de la cultura investigativa, favoreciendo la producción académica.

Para lograr esos propósitos en la sede de Guayaquil, el colectivo de profesores del CLEA se conformó con un coordinador y docentes escogidos de cada una de las carreras de la Universidad. La mayoría de los profesores poseen formación especializada en el Idioma Español y la Literatura en varios casos. Otros docentes cuentan con un buen desarrollo de las habilidades lingüísticas, así como excelentes relaciones interpersonales y ferviente deseo de servir a la comunidad educativa.

\section{Metodología}

El colectivo del CLEA elaboró un programa de trabajo, con diversas actividades en su etapa inicial para promover las primeras acciones. En ese momento se efectuaron foros con el propósito de dar a conocer la existencia del centro, mediante la explicación de sus objetivos y su contenido de trabajo. En una segunda etapa se desarrollaron conversatorios y veladas literarias, así como se ofrecieron cursos de capacitación docente sobre motivación para el empleo de estrategias de lectura y escritura, redacción gramatical y científica. Posteriormente, se creó el "Curso Virtual de Actualización de Conocimientos" sobre lengua castellana para complementar procesos de formación académica en la universidad. La última propuesta fue la realización del Aula Virtual de la asignatura de Técnicas de Expresión Oral y Escrita, la cual incorporará todos los elementos del currículo, incluyendo el seguimiento y las evaluaciones para su aprobación.

Como experiencia novedosa se puede mencionar que, a partir de la necesidad de fortalecer las competencias de lectura y escritura desde el proceso pedagógico universitario, la aplicación de un conjunto de acciones podría influir positivamente sobre los estudiantes, docentes y personal administrativo. Las principales actividades realizadas incluyeron:

\section{Foros}

La primera actividad que realizó el Centro de Lecto Escritura Académica fue el Foro "Conciencia lingüística" dirigido a particularmente a los estudiantes y a la comunidad educativa en general. Fueron 10 foros, pero con el mismo contenido. La asistencia 
sobrepasó los 700 asistentes y su propósito fue difundir la existencia del Centro, sus objetivos y las actividades que iba a realizar.

Los docentes, integrantes del Centro, en sus horas de clase conducían a los estudiantes al auditorio para que participen de esta actividad que en realidad fue un despertar del "conocimiento explícito acerca de la lengua, la percepción y la sensibilidad conscientes al aprender la lengua, al enseñarla y al usarla" (Centro Virtual Cervantes., 2017).

Los profesores informaron a los presentes de forma oral y con apoyo de diapositivas todo lo concerniente acerca de la creación del Centro y sus responsabilidades. Asimismo, respondieron las inquietudes e interrogantes de los asistentes. El coordinador del foro elaboró una conclusión, la misma que fue publicada más tarde y masivamente en las redes informáticas de la universidad.

\section{Conversatorios, encuentros, tertulias y veladas literarias}

Una vez que se aprende a leer y escribir gracias al conocimiento de la normativa y la práctica de un idioma, no hay que olvidar que estas dos habilidades continúan aprendiéndose y perfeccionándose en el resto de nuestras vidas. La literatura viene a ser la continuación del conocimiento de la lengua castellana ya que es la manifestación artística basada en el uso del lenguaje. Otras interpretaciones señalan que es el estudio de los escritores con sus respectivas obras literarias, muchas de las cuales han sido tomadas como ejemplos o modelos a seguir.

Las experiencias recogidas por quienes se dedican a la docencia en esta asignatura, en este caso, los autores de este artículo, estiman que la literatura nos permite conocer cómo ama y cómo piensa el hombre en una época determinada. Entonces, en una Universidad como la UPS donde la mayoría de las Carreras son técnicas, y casi no se imparten asignaturas sociales, resulta imprescindible realizar actividades que permitan el conocimiento del arte en las letras, es una oportunidad para comprender los sentimientos ajenos, encontrar elementos comunes y darnos cuenta que son muchos los referentes de identificación que tenemos en contraposición con aquellos que nos diferencian. De ahí que estos espacios de lectura y análisis de obras literarias son momentos muy oportunos para conocer y estudiar el pensamiento de grupos humanos de otras épocas, esto viene a ser un proceso educativo que, de alguna manera, nos lleva a una educación intercultural.

Es importante reconocer, que los docentes tenían una duda racional respecto a la aceptación de las actividades literarias por parte de los estudiantes, puesto que ellos son estudiantes de carreras técnicas que utilizan herramientas y equipos tecnológicos con un propósito práctico. Un fin distinto es el de la literatura que es el proporcionar emociones y goces estéticos apreciando el buen uso de la lengua castellana.

Con este antecedente y con mucha prolijidad e imaginación, se planificaron eventos como "El Encuentro con la literatura clásica" para analizar los cuentos del escritor norteamericano Edgar Allan Poe. La actividad tuvo varios objetivos: 
- Promover la lectura de las obras literarias consideradas modelos por su calidad y porque forman parte del patrimonio cultural de la humanidad.

- Fortalecer la competencia comunicativa en cuanto a la expresión oral.

- Desarrollar la lectura crítica de los estudiantes salesianos de todas las carreras que se interesaron en participar en el evento.

Previamente, ellos fueron incentivados en las aulas por los docentes, integrantes del CLEA, quienes explicaron la importancia del autor y les hicieron llegar todo el material necesario como: datos biográficos, videos, clasificación de sus obras y argumentos de algunas seleccionadas. La modalidad escogida fue el conversatorio. Son fundamentales las prácticas previas a la actividad y las observaciones de los docentes ya que, en este caso, se convirtieron en una verdadera revisión de la lectura.

Por ejemplo, se repasaron sus procesos, notándose que algunos de los estudiantes eran lectores en situación de aprendizaje; otros tomaron la lectura como una herramienta de análisis; observándose, además y durante los ensayos, la incorporación de registros lingüísticos propios de la expresión literaria. Asimismo, el momento sirvió para corregir aspectos verbales como la claridad, la concisión, la precisión, etc. y aspectos de la voz como la pronunciación, el tono, volumen, velocidad, fluidez, pausas. También se hizo hincapié en el empleo correcto de la voz para enfatizar los puntos que se desean resaltar y así mantener la atención del público.

En el momento del evento, estaban reunidos más de 30 estudiantes-participantes y más de 100 compañeros y docentes como espectadores. Los alumnos habían adornado el auditorio con los propios trabajos los cuales consistían en hermosos dibujos, pequeñas arquitecturas y esculturas referentes a la vida del escritor, así como a su obra literaria. Estas muestras artísticas no solo significaron una exaltación al autor y a su producción literaria, sino que también representó un encuentro con las bellas artes. Seguidamente, los estudiantes realizaron lecturas y análisis de fragmentos seleccionados y contestaron una ronda de preguntas de los presentes tanto sobre la vida del autor como del contenido de la obra. Esta parte fue muy dinámica ya que aportó riqueza al conversatorio y este, a su vez, vigorizó las discusiones. El moderador, un profesor de la universidad, siempre estuvo atento al desenvolvimiento del conversatorio señalando pausas, tiempo o respeto a las intervenciones. Al final, las reflexiones fueron complementadas por los docentes que integran el CLEA quienes consensuaron las exposiciones y comentarios a fin de que no queden sueltas o aisladas ideas o análisis valiosos, añadiendo que estos eventos garantizan el acceso, la construcción y el ejercicio de la cultura como condición para el desarrollo humano.

Otro evento literario que se realizó fue la "Velada literaria y musical sobre la Vida y Obras de Mario Benedetti" con la participación de 6 o 10 estudiantes por cada una de las carreras y más de 100 asistentes entre profesores y otros alumnos. Al igual que en el conversatorio anterior, se dotó a los estudiantes de todo el material necesario; las actividades previas o lo ensayos fueron de gran significación ya que permitieron revisar la lectura inferencial, es decir, interpretar todo aquello que el autor quiere comunicar, pero que en ocasiones no lo dice o escribe explícitamente. En lo que respecta, a la lectura crítica se valoró y se juzgó tanto el contenido de la obra planteada por el autor como las inferencias o relaciones que se pueden establecer a partir de lo que aparece en el texto producido, pero con 
argumentaciones. Estas surgen de acuerdo a los conocimientos, experiencias de vida, a la escala de valores y a la cultura a la cual pertenecen los lectores que en esta oportunidad fueron los estudiantes y cuyas aportaciones fueron valoradas según estos parámetros. En la lectura de los poemas, por ejemplo, los amorosos, se relacionaron las emociones propias con las del texto leído, se identificaron los temas y características de ellos; se revisaron los rasgos literarios (recursos estilísticos) y la intención del autor. Se ensayaron las declamaciones y las interpretaciones musicales de los poemas como "Te quiero" y "Hagamos un trato" de Mario Benedetti.

Como toda velada, el evento se realizó por la noche en un auditorio acogedor y con un ambiente lleno de cordialidad y alegría. Los estudiantes de todas las carreras de la universidad en compañía de los profesores del CLEA, compartieron -sin grandes formalismos- reflexiones sobre la vida y obra del autor. Entre comentarios y opiniones, se declamaron poemas seleccionados de Benedetti o se interpretaba su poesía musicalizada. Fue una reunión para aprender, conversar y divertirse.

Los objetivos que se persiguieron con la velada literaria fueron:

- Motivar a los estudiantes para que se conviertan en lectores asiduos de las obras literarias de los grandes autores.

- Fortalecer las habilidades lingüísticas, especialmente la lectura en sus niveles inferencial y crítica valorativa ya que conducen al análisis de libros.

- Reforzar la comprensión de escritos que se refieran a los acontecimientos del diario vivir como notas periodísticas, publicidades y la interpretación de los mensajes ocultos en cualquier discurso.

Como resultado de los eventos literarios se resaltan las opiniones de los estudiantes, quienes comentaban que estos momentos les había servido para comprender lo grandioso e influyente que es la lengua castellana. Ya sea una palabra o muy pocas, así como las expresiones idiomáticas les habían dejado infinidad de sentimientos, pensamientos, los mismos que les hacían surgir nuevas sensaciones o ideas. Los estudiantes refirieron que con la literatura se ubicaban plenamente en este tiempo y podían imaginarse como serían los tiempos pasados en iguales circunstancias y contextos. Lo cierto es que el "arte de la palabra" y la lectura de las obras literarias dejan en los sentidos y en el espíritu la complacencia de vivir para uno y los demás. De ahí que la literatura es una disciplina humana, al desarrollar los valores morales, cívicos y ecológicos en quienes la estudian.

\section{Entrevistas a escritores}

El Centro de Lecto Escritura Académica dedica espacios a las entrevistas para relievar la trayectoria de personajes que hacen literatura y que, en este caso, representan a la poesía y la narrativa ecuatoriana de los últimos tiempos. Se realizaron entrevistas a poetas guayaquileños, ecuatorianos y docentes de la Universidad con presencia en casas editoras y en círculos poéticos de América y Europa. Parte de sus obras están traducidas a más de diez idiomas. Los diálogos se centraron en sus trabajos literarios y, también para conocer la revitalización del lenguaje y la evolución de la Literatura en América Latina. Las 
entrevistas se difundieron por el UPS Channel (canal online) y las demás redes informáticas de la universidad.

Las conversaciones con escritores es otra forma de difusión de la literatura a través de las cuales se pone de manifiesto las personalidades de los literatos, la divulgación de sus escritos, así como las características del mundo lector actual. Estas entrevistas sirven, a los docentes de las asignaturas relativas al idioma español, como tema de clase para sus alumnos, para el análisis y comentario de la personalidad de los autores, la profundidad de sus respuestas, la riqueza del léxico y las particularidades de su producción literaria.

\section{Cursos de capacitación docente (CLEA, 2017)}

Varias acciones de capacitación fueron ejecutadas bajo la dirección del CLEA. En ese contexto se incluye el Curso de Redacción Académica impartido a más de 60 docentes de la asignatura Técnicas de Expresión Oral y Escrita, así como otros interesados. En el primer día del curso, los docentes realizaron una prueba de diagnóstico para detectar las debilidades en cuanto a la comprensión y producción de textos escritos, la misma que una vez revisada, fue entregada para su conocimiento, solicitándoles que la guarden para que sea comparada con otras evaluaciones. La planificación del curso fue la siguiente:

\begin{tabular}{|c|c|}
\hline Primer día & $\begin{array}{l}\text { Motivación } \\
\text { Tipos de Texto } \\
\text { Propiedades textuales: Coherencia y cohesión }\end{array}$ \\
\hline Segundo día & $\begin{array}{l}\text { Concordancia } \\
\text { Uso de Gerundios } \\
\text { Verbos Irregulares } \\
\text { Vicios del Lenguaje }\end{array}$ \\
\hline Tercer día & $\begin{array}{l}\text { Estructura del párrafo } \\
\text { Clases de párrafo }\end{array}$ \\
\hline Cuarto día & $\begin{array}{l}\text { Acentuación } \\
\text { Puntuación } \\
\text { Actualizaciones de la RAE } \\
\text { Abreviaturas } \\
\text { Errores comunes }\end{array}$ \\
\hline Quinto día & $\begin{array}{l}\text { Talleres } \\
\text { Examen }\end{array}$ \\
\hline
\end{tabular}

Al final del estudio, los trabajos en clase, así como las evaluaciones demostraron un mejoramiento evidente en la sintaxis y la semántica en los resúmenes y comentarios escritos de los docentes. Se observó que comunicaban sus propias ideas o de los autores de manera coherente y con escasos errores sintácticos. En cuanto a la ortografía, se registró un $95 \%$ de aciertos, no así en la puntuación que alcanzó solo el $80 \%$.

El último nivel que se evaluó fue el razonamiento lógico y la argumentación. Se reconoció que entre el $80 \%$ y el $85 \%$ mostraron coherencia y cohesión en sus ideas como también capacidad argumentativa. De lo expuesto se deduce, que las motivaciones empleadas en cada clase evidenciaron su eficacia; que cuando los docentes de las distintas áreas del conocimiento se muestran interesados en alcanzar un mayor desarrollo de las competencias de la 
lengua materna, contribuyen al crecimiento de los niveles de lectura y escritura en los estudiantes. Aún más, si se considera este accionar como una práctica didáctica y cotidiana en los ciclos o niveles educativos y para todos los saberes disciplinares.

Otra propuesta fue el curso "Motivación de Lectura y Escritura Académica", con la presencia de 123 profesores de todas las carreras. El objetivo del curso se concentró en contribuir al desarrollo de habilidades didácticas de los docentes para la motivación de la lectura y escritura académica. Los tutores fueron profesores de la universidad e integrantes del CLEA.

Los temas y la planificación del curso se organizaron siguiendo el siguiente esquema:

\begin{tabular}{ll}
\hline $\begin{array}{l}\text { Primer } \\
\text { día }\end{array}$ & $\begin{array}{l}\text { Introducción: Importancia de la lecto escritura en la formación del profesional universitario. } \\
\text { Las habilidades fundamentales de la lengua materna. Aspectos generales de la Didáctica de lecto } \\
\text { escritura. }\end{array}$ \\
\hline $\begin{array}{l}\text { Segundo } \\
\text { día }\end{array}$ & Concepciones sobre lectura. Tipos. Estrategias de comprensión lectora. Promoción de la lectura en la \\
Tercer & $\begin{array}{l}\text { Universidad. } \\
\text { día }\end{array}$ \\
$\begin{array}{l}\text { Concepciones sobre escritura. Proceso de la escritura académica. Escritura argumentativa. } \\
\text { día } \\
\text { Quinto } \\
\text { día }\end{array}$ & $\begin{array}{l}\text { Propiedades textuales. Promoción de la escritura. } \\
\text { Lallectura y escritura de textos científicos. Las nuevas tecnologías y la lectoescritura científica. }\end{array}$ \\
\hline
\end{tabular}

La metodología del curso incluyó el desarrollo de conferencias, debates, trabajo en equipos, así como trabajo colaborativo y autónomo. Esas formas de organización del proceso docente, posibilitan la socialización y asimilación del contenido de forma cooperativa. Una atención preferente se otorgará a la vinculación teoría - práctica. El curso se extendió por 40 horas, distribuidos en 20 horas de trabajo de autónomo con actividades enviadas por el tutor utilizando el entorno virtual de aprendizaje de la universidad y 20 horas de trabajo presencial. La variedad de medios empleados se completó con el uso de computadora, video beam, pizarra, papelógrafos, marcadores, libros y publicaciones periódicas.

\section{Curso virtual de actualización de conocimientos}

Un estadio superior ha sido la creación del GIE- CLEA (Grupo de Innovación Educativa del Centro de Lecto Escritura Académica), que tiene entre sus acciones principales el desarrollo del "Curso Virtual de Actualización de Conocimientos" de gramática castellana. Su propósito se dirige a complementar procesos de formación académica en la universidad, sobre todo de los estudiantes que, habiendo culminado sus estudios en la universidad, no se han graduado por diversas razones y estén interesados en terminarlos.

Esta actualización permitirá al estudiante alcanzar el dominio de las macro habilidades de la lengua materna y con ellas abundantes destrezas en la expresión oral y escrita. Ello les permite desenvolverse fluidamente en charlas, comentarios, críticas constructivas y argumentaciones. Todo ello incide en el acceso a nuevos empleos, permitiendo adaptarse a los posibles contextos laborales y coadyuvar a la integración social.

\section{Aula virtual de la asignatura de comunicación oral y escrita}


Otra actividad del CLEA es la implementación de un aula virtual, utilizando las posibilidades que ofrece la educación a distancia en la asignatura de Comunicación oral y escrita. Esta nueva forma de enseñar, que ya se utiliza en muchas universidades a nivel mundial y en algunas otras entidades dedicadas a la ayuda y apoyo de los estudiantes, significará en nuestra institución un auxilio para quienes trabajan. Toda enseñanza, mediada por las tecnologías de la información y la comunicación aporta recursos sugerentes y estimulantes en el aprendizaje. Esto repercute en el manejo y comprensión de los contenidos y a la final contribuye a disminuir la deserción de los alumnos.

\section{Un espacio de asesoría linguiística o corrección idiomática}

El Centro de Lecto Escritura Académica se ha constituido en muy poco tiempo en un espacio de asesoría para el buen manejo de la lengua castellana, tanto para los estudiantes como los docentes. Los profesionales encargados de realizar estas actividades son los propios profesores que integran el centro, quienes se encargan de corregir textos, revisar resúmenes, oficios, solicitudes, informes, desarrollo y análisis de temas, críticas, argumentaciones, etc. Esta revisión se concreta en el uso correcto de la coherencia y la cohesión, como propiedades textuales.

\section{Análisis y resultados}

\section{Resultados de la prueba diagnóstico}

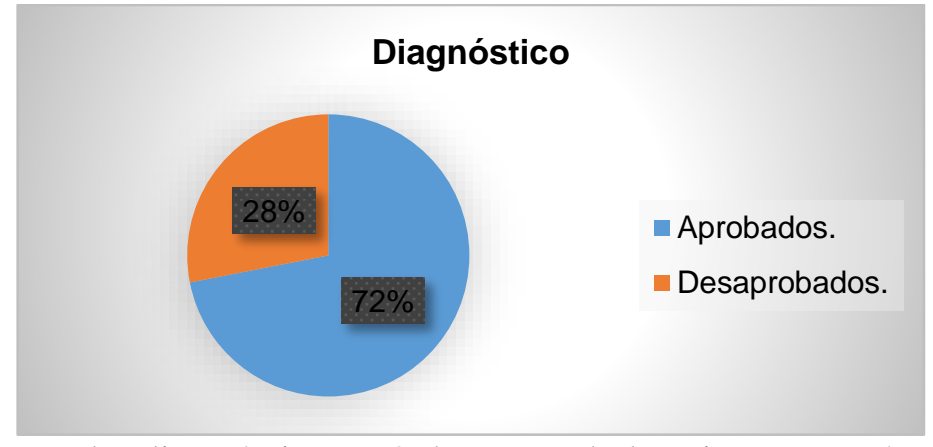

Se realizó una prueba diagnóstica a 60 docentes de la asignatura Técnicas de Expresión Oral y Escrita. El objetivo de la prueba fue detectar las debilidades que tenían los docentes en cuanto a la comprensión y producción de textos escritos. Esta prueba fue revisada y evaluada, y los resultados, así como comentarios sobre sus dificultades fueron entregados a los docentes antes de comenzar el curso.

\section{Resultados de la entrevista}




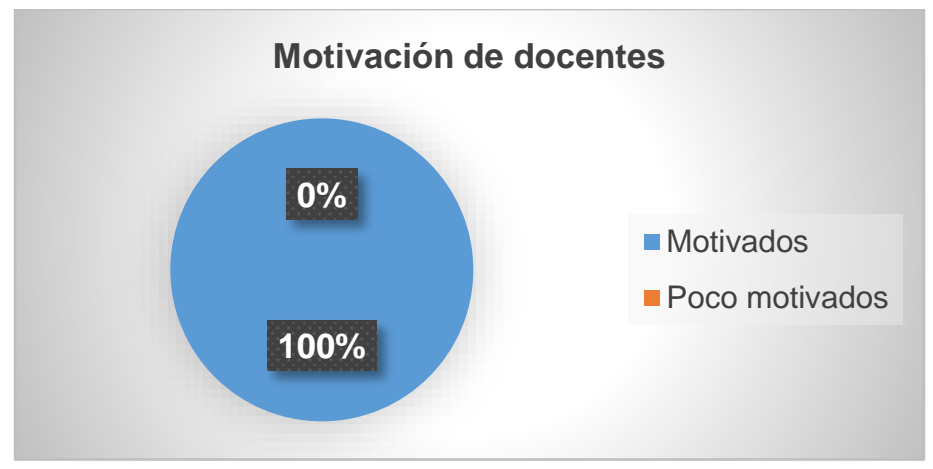

Se realizó además una breve entrevista a 123 profesores de todas las carreras. El objetivo de la misma fue conocer el nivel de conocimientos y el interés de estos docentes en el curso para contribuir al desarrollo de habilidades didácticas de los docentes para la motivación de la lectura y escritura académica. Esta entrevista arrojó un alto índice de profesores motivados en la participación en el curso.

\section{Resultados de las evaluaciones de los cursos}

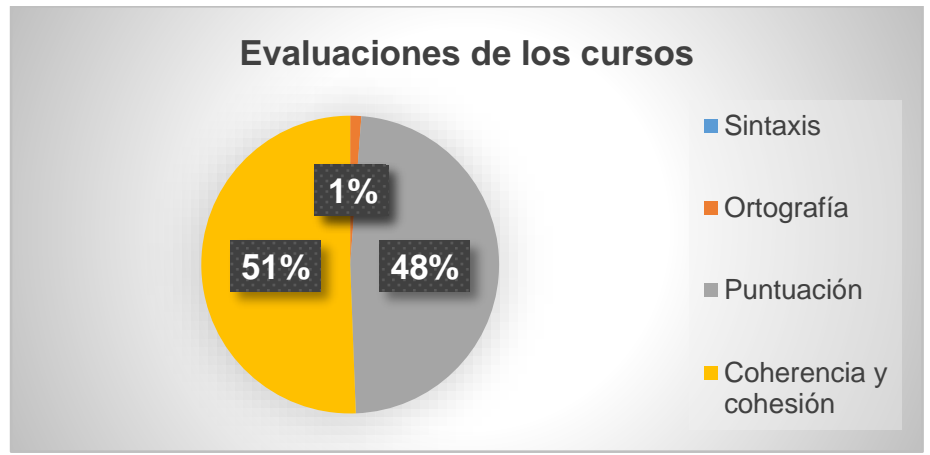

Al final de los cursos, se realizaron algunas evaluaciones que demostraron un mejoramiento evidente en la sintaxis y la semántica en los resúmenes y los comentarios escritos de los docentes. Se observó que comunicaban sus propias ideas o de los autores de manera coherente y con escasos errores sintácticos. En cuanto a la ortografía, se registró un $95 \%$ de aciertos, no así en la puntuación que alcanzó solo el 80\%. El último nivel que se evaluó fue el razonamiento lógico y la argumentación. Se reconoció que entre el $80 \%$ y el $85 \%$ mostraron coherencia y cohesión en sus ideas como también capacidad argumentativa.

\section{Discusión}

Se observó de manera general que los docentes estaban altamente motivados para la participación activa en los cursos. En el diagnóstico pudieron percatarse de los problemas que aún tenían y que podían resolver o atenuar con la impartición de estos cursos. La participación en las entrevistas fue muy buena. Los docentes pudieron expresar sus ideas, expectativas y opiniones acerca de diferentes temas de la Lecto-escritura y las habilidades lingüísticas en general. La participación de los docentes se mantuvo en un $100 \%$ en todos los cursos impartidos, y todos cooperaron en las diferentes evaluaciones, tareas y debates allí propiciados. A diferencia 
de otros encuentros, este ha sido muy enriquecedor, ya que se pudo conocer con exactitud los temas de interés de los docentes así como sus dificultades académicas y la forma de resolverlas.

\section{Conclusiones}

En resumen, puede afirmarse que las actividades efectuadas y descritas, han posibilitado alcanzar peldaños superiores en la cultura para la lecto escritura. Ello se percibe en la satisfacción expresada por los participantes en las acciones ejecutadas. La puesta en práctica del cronograma de trabajo posibilitó la participación de un alto porcentaje de estudiantes y docentes de la sede involucrados en las actividades para promover la lectura y la escritura de la comunidad educativa. El saldo resultó una mejora notable en el dominio del idioma de quienes forman parte de la institución y un estímulo a la integración social a través del lenguaje. Se evidenció, además, que la creación de estructuras dentro de las instituciones de Educación Superior para fomentar la lectura y la escritura académica, propicia la ejecución de un conjunto de acciones estratégicas en función de lograr la motivación hacia los complejos procesos asociados al idioma. Ello es un factor válido de emprendimiento educativo, a partir de la articulación de procedimientos didácticos adecuadamente diseñados y ejecutados. Esas acciones estratégicas incluyen la planificación y ejecución de diversas formas de organización como foros, conversatorios, encuentros, tertulias y veladas literarias, entrevistas a escritores, asesoría lingüística, cursos de capacitación docente, cursos y aulas virtuales de comunicación.

La ejecución de un cronograma de trabajo dirigido por los integrantes del CLEA, demostró la importancia de los procesos de la lecto escritura en el incremento de la motivación lectora en los educandos. A su vez, el empleo de variadas formas de incentivo y la aplicación de peculiares estrategias de aprendizaje permitieron mejorar las habilidades lingüísticas de los estudiantes. Toda esta cadena de procedimientos logrará un mayor acceso al mundo del conocimiento, al perfeccionamiento de su formación profesional y a la apertura a un mundo multicultural y plurilingüista como futuros emprendedores en diversos campos de las tecnologías y las humanidades.

\section{Recomendaciones}

Se debe continuar con la promoción e impartición de cursos que respondan a las demandas y exigencias de los docentes y estudiantes involucrados en el proceso de enseñanzaaprendizaje de la lengua española, la lecto escritura y el desarrollo de las habilidades lingüísticas de manera general. Se debe además dar a conocer los resultados de estos cursos para promover la participación de más docentes y personal especializado interesado en el mejoramiento de la enseñanza y el aprendizaje de la lengua española.

\section{Bibliografía}

Alonso, F. (2007) "La importancia de la literatura en la escuela y en la casa". Alicante: Biblioteca Virtual Miguel de Cervantes, 2007

Alonso, Fernando. (2017) La importancia de la literatura en la escuela y en la casa. Tomado de: http://www.cervantesvirtual.com/obra-visor/la-importancia-de-la-literatura-en-la-escuelay-en-la-casa-0/html/016800a8-82b2-11df-acc7-002185ce6064_2.html\#I_0_ 
Azpillaga Larrea, Verónica. (2011) Formación de profesorado en educación intercultural desde la reflexión sobre la acción. En: El poder de la comunicación en una sociedad globalizada. Almería. Tomado de: https://books.google.com/books?isbn=8482405462

Cabo Aseguinolaza, F. y María do Cebreiro. Manual de la teoría de la literatura, 2006, pág. 71

Cabo Aseguinolaza, Fernando. Cebreiro Rábade-Villar, María do (2006). Manual de teoría de la literatura. Madrid-España. Editorial Castalia.

Carlino, P. (2005). Escribir, leer y aprender a leer en la universidad. Una introducción a la alfabetización académica. Buenos Aires: Fondo de Cultura Económica de Argentina. P. 5

Carlino, Paula (2005). Escribir, leer y aprender en la universidad. Una introducción a la alfabetización académica. Buenos Aires: Fondo de Cultura Económica. ISBN: 9505576536. Selección de textos tomados de la Introducción. Comité Editorial de UniPluri/Versidad. Tomado www.ub.edu.ar/.../Carlino_Escribir_leer_y_aprender_en_la_Universidad.pdf

Centro de Lecto-Escritura Académica UPS. (2013) Tomado de: http://lectoescritura.blog.ups.edu.ec

Centro Virtual Cervantes. (2017) Enseñanza. Tomado de: https://cvc.cervantes.es

CLEA. (2017) Educación online. México-Bogotá. Tomado de: https://clea.edu.mx/portal/

Cobo, Cristóbal. (2012) Competencias para empresarios emprendedores: contexto europeo. Tomado de: https://www.oitcinterfor.org/sites/default/files/file_publicacion/comp_empre_cobo.pdf

González, P. L. y D. Hernández. Capacitación Profesoral y Didáctica Universitaria. INNOVA Research Journal 2016, Vol 1, No. 11, 30-41. ISSN 24779024.http://www.journaluidegye.com/magazine/index.php/innova/article/view/60/101. Autor

González, P. L. y D. Hernández. Educación para el emprendimiento/Education for entrepreneurship Education. Repositorio Científico Institucional, Universidad de Pinar del Río "Hermanos Saíz Montes de Oca”, Cuba. http://rc.upr.edu.cu/handle/DICT/902 // Revista científico pedagógica Mendive, Vol. 13, Núm. 4 pp. 418-423. 2015. http://mendive.upr.edu.cu/index.php/MendiveUPR/article/view/782.

González, P. L. y M. I. Salgado. (2017) Inclusión educativa, capacitación profesional y motivación por la lectura. III Congreso Internacional de Ciencias Pedagógicas, Guayaquil, Ecuador. Instituto Superior Tecnológico Bolivariano de Tecnología, UNESCO. 20 al 22 de abril de 2017. CD de Memorias. ISBN 978-9942-17-027-9. Autor 
Proyecto Avanza @: Competencias para la productividad de las Mipymes centroamericanas en la economía del conocimiento y la innovación. Fundación Omar Dengo-IDRCOIT/CINTERFOR. "Competencias para empresarios emprendedores: contexto europeo. Responsable: Cristóbal Cobo Fecha de elaboración: 24/01/2012 Versión. 0.4”.

Rodríguez, Iván Diego y José Antonio Vega Serrano. (2015) La educación para el emprendimiento en el sistema educativo español. Año 2015. Estudio Redie. Ministerio de educación, cultura y deporte. Tomado de: www.sel-gipes.com/.../2016_-_ue__entrepreneurship_education_at_school_in_europ...

Universidad Politécnica Salesiana del Ecuador. Instructivo del CLEA. Documento digital.

Vazquez, Alicia, María del Carmen Novo, et al. (2010) Lectura, escritura y apendizaje interdisciplinar. Jornadas sobre lectura, escritura y apendizaje interdisciplinar. 9 y 10 de septiembre de 2010. Universidad Nacional de Río Cuarto. Facultad de Ciencias Humanas. Editora Unirío. Tomado

de: https://www.unrc.edu.ar/unrc/digtal/libro_jornadas_unesco_unrc_2010.pdf 